\title{
The Dual Action of Fractional Multi Time Hamilton Equations
}

\author{
Dumitru Baleanu • Ali Khalili Golmankhaneh • \\ Alireza Khalili Golmankhaneh
}

Received: 21 January 2009 / Accepted: 11 May 2009 / Published online: 22 May 2009

(C) The Author(s) 2009. This article is published with open access at Springerlink.com

\begin{abstract}
The fractional multi time Lagrangian equations has been derived for dynamical systems within Riemann-Liouville derivatives. The fractional multi time Hamiltonian is introduced as Legendre transformation of multi time Lagrangian. The corresponding fractional Euler-Lagrange and the Hamilton equations are obtained and the fractional multi time constant of motion are discussed.
\end{abstract}

Keywords Riemann-Liouville derivative and fractional Euler Lagrange equations · Constant of fractional motion · Dual action

\section{Introduction}

Derivatives and integrals of fractional order have found many applications in recent studies in mechanics and physics. For example, in chaotic dynamics, quantum mechanics, plasma physics, anomalous diffusion, and so many fields of physics $[8,10,11,15,17,18,24$, 29, 31]. Especially in mechanics Riewe has shown that Lagrangian involving fractional time derivatives leads to equation of motion with non conservative classical derivatives such

D. Baleanu $(\bowtie)$

Department of Mathematics and Computer Science, Çankaya University, 06530 Ankara, Turkey

e-mail: dumitru@cankaya.edu.tr

D. Baleanu

Institute of Space Sciences, PO Box, MG-23, 76900, Magurele, Bucharest, Romania

Alireza K. Golmankhaneh

Department of Physics, University of Pune, Pune 411007, India

e-mail: alireza@physics.unipune.ernet.in

Alireza K. Golmankhaneh · Ali K. Golmankhaneh

Department of Physics, Islamic Azad University-Uromia Branch, PO Box 969 Uromia, Iran

Ali K. Golmankhaneh

e-mail: ali.khalili.gol@gmail.com 
as friction [22, 23]. Motivated by this approach many researchers have explored this area giving new insight into this problem [4, 5, 12-14, 16, 19-21, 25-27, 30]. Agrawal has presented fractional Euler-Lagrange equation in Riemann-Liouville derivatives [1]. Further fractional single time Hamiltonian formulation has been developed by Baleanu and coworkers $[2,3]$. Classical multi time Hamilton equations were firstly introduced by de Donder in 1936 [7]. In recent years multi-time Hamiltonian and its applications become an interesting area for authors $[6,28]$. In this paper we generalize classical multi time Lagrangian and Hamiltonian to fractional multi time motion. We show that the classical dual action can be defined in the fractional case but the connection between the periodic solution of multitime Hamiltonian and the periodical critical points of the dual action collapses [6]. The paper is organized as follows: Sect. 2 has three subsections. The first subsection presents briefly some basic definitions of Riemann-Liouville and Caputo derivatives, the remaining ones contain the formalism of single time fractional Lagrangian and Hamiltonian. In Sect. 3 we introduce multi time fractional Lagrange equations. Section 4 work out the multi time fractional Hamilton equations. In Sect. 5 we have studied fractional conservation laws. Fractional Noether theorem is offered in the multi time fractional mechanics and we arrive at the standard cases. Further in Sect. 6 suitable Lagrangian for fractional dual action has been derived. In Sect. 7 the Euler-Lagrange equations of the fractional dual action and corresponding multi time Hamilton equations is presented and the connection between the periodic solution of multi-time Hamiltonian and the periodical critical points of the dual action in the fractional mechanics is discussed. Section 8 is devoted to our conclusions.

\section{Basic Tools}

\subsection{Fractional Derivatives}

In this section we briefly present the definition of the left and the right fractional derivatives of Riemann-Liouville as well as Caputo [28]. The left Riemann-Liouville fractional derivative is defined as

$$
{ }_{a} D_{t}^{\alpha} f(t)=\frac{1}{\Gamma(n-\alpha)}\left(\frac{d}{d t}\right)^{n} \int_{a}^{t} \frac{f(\tau)}{(t-\tau)^{\alpha+1-n}} d \tau,
$$

and the right Riemann-Liouville fractional derivative,

$$
{ }_{t} D_{b}^{\alpha} f(t)=\frac{1}{\Gamma(n-\alpha)}\left(-\frac{d}{d t}\right)^{n} \int_{t}^{b} \frac{f(\tau)}{(\tau-t)^{\alpha+1-n}} d \tau,
$$

where the order $\alpha$ fulfills $n-1 \leq \alpha<n$ and $\Gamma$ represent the gamma function. An alternative definition of Riemann-Liouville fractional derivative called Caputo derivative that introduced by Caputo in 1967. The left Caputo derivative defined as

$$
{ }_{a}^{C} D_{t}^{\alpha} f(t)=\frac{1}{\Gamma(n-\alpha)} \int_{a}^{t}(t-\tau)^{n-\alpha-1}\left(\frac{d}{d \tau}\right)^{n} f(\tau) d \tau,
$$

and the right Caputo Fractional Derivative

$$
{ }_{t}^{C} D_{b}^{\alpha} f(t)=\frac{1}{\Gamma(n-\alpha)} \int_{t}^{b}(\tau-t)^{n-\alpha-1}\left(-\frac{d}{d \tau}\right)^{n} f(\tau) d \tau,
$$


where the order $\alpha$ satisfies $n-1 \leq \alpha<n$. The Riemann-Liouville derivative of constant isn't zero, although Caputo derivative of a constant is zero.

\subsection{Single Time Fractional Lagrangian}

From the mathematical point of view both left and right derivatives could appear within a fractional Lagrangian of a system, $L\left(t, q^{i}(t),{ }_{a} D_{t}^{\alpha} q^{i},{ }_{t} D_{b}^{\beta} q^{i}\right), i=1, \ldots, n$ where $\left(q^{i}(t)\right)=$ $\left(q^{1}(t), q^{2}(t), \ldots, q^{n}(t)\right)$, but in some physics problems, if $t$ denotes the time variable, the right fractional derivatives of $q^{i}(t)$ is interpreted as future state of the process $q^{i}(t)$. For this reason in those problems the right derivative is usually neglected in application when the present state of the process does not depend on the result of the future or at least we consider this sort of problems. Let us consider a Lagrangian as $L\left(t, q^{i},{ }_{a} D_{t}^{\alpha} q^{i}\right)$. The Lagrangian $L: R \times R^{2 n} \rightarrow R,\left(t, q^{i},{ }_{a} D_{t}^{\alpha} q^{i}\right) \rightarrow L\left(t, q^{i},{ }_{a} D_{t}^{\alpha} q^{i}\right)$ and $\left({ }_{a} D_{t}^{\alpha} q^{i}\right)=$ $\left.{ }_{a} D_{t}^{\alpha} q^{1}(t),{ }_{a} D_{t}^{\alpha} q^{2}(t), \ldots,{ }_{a} D_{t}^{\alpha} q^{n}(t)\right)$ produces the single time fractional Euler-Lagrange equation given below

$$
\frac{\partial L}{\partial q^{i}}+{ }_{t} D_{b}^{\alpha} \frac{\partial L}{\partial_{a} D_{t}^{\alpha} q^{i}}=0, \quad i=1,2, \ldots, n .
$$

\subsection{Single Time Fractional Hamilton Equations}

The Lagrangian $L$ defines the diffeomorphism, ${ }_{a} D_{t}^{\alpha} q^{i}(t) \rightarrow p_{i}=\frac{\partial L}{\partial_{a} D_{t}^{\alpha} q^{i}}$ called the Legendre transformation. The Legendre transformation of the Lagrangian $L$ is the Hamiltonian $H$. If $p_{i}=\frac{\partial L}{\partial_{a} D_{t}^{\alpha} q^{i}}$ be fractional $i$-momentum we obtain the fractional multi time Hamiltonian as follows

$$
H\left(t, q^{i}, p_{i}\right)={ }_{a} D_{t}^{\alpha} q^{i} \frac{\partial L}{\partial_{a} D_{t}^{\alpha} q^{i}}-L .
$$

Calculating the total differential of this Hamiltonian we obtain

$$
d H={ }_{a} D_{t}^{\alpha} q^{i} d p_{i}-\frac{\partial L}{\partial q^{i}} d q^{i}-\frac{\partial L}{\partial t} d t .
$$

By making use of

$$
d H=\frac{\partial H}{\partial t} d t+\frac{\partial H}{\partial p_{i}} d p_{i}+\frac{\partial H}{\partial q^{i}} d q^{i},
$$

and by using the fractional Euler-Lagrange, we get the fractional Hamilton equations as

$$
\begin{aligned}
& \frac{\partial H}{\partial t}=-\frac{\partial L}{\partial t}, \\
& \frac{\partial H}{\partial p_{i}}={ }_{a} D_{t}^{\alpha} q^{i}, \\
& \frac{\partial H}{\partial q^{i}}={ }_{t} D_{b}^{\alpha} p_{i} .
\end{aligned}
$$

It is worth to mention that for $\alpha \rightarrow 1$ equations reduce to classical Hamilton equations. The fractional Hamiltonian isn't a constant of motion even though the Lagrangian doesn't depend on the time explicitly. This can be observed by making use of

$$
\frac{d H}{d t}=\frac{\partial H}{\partial p_{i}} \frac{d p_{i}}{d t}+\frac{\partial H}{\partial q^{i}} \frac{d q^{i}}{d t},
$$


and by substituting the values of partial derivatives of Hamiltonian from (9). As a result we obtain that

$$
\frac{d H}{d t}={ }_{a} D_{t}^{\alpha} q^{i} \frac{d p_{i}}{d t}+{ }_{t} D_{b}^{\alpha} p_{i} \frac{d q^{i}}{d t} \neq 0
$$

Using the symplectic structure

$$
J=\left(\begin{array}{cc}
0 & \delta_{j}^{i} \\
\delta_{j}^{i} & 0
\end{array}\right),
$$

we may write the Hamilton equations in the matrix form

$$
-\left(\begin{array}{cc}
0 & \delta_{j}^{i} \\
\delta_{j}^{i} & 0
\end{array}\right)\left(\begin{array}{c}
{ }_{a} D_{t}^{\alpha} q^{j} \\
{ }_{t} D_{b}^{\alpha} p_{j}
\end{array}\right)+\left(\begin{array}{c}
\frac{\partial H}{\partial q^{i}} \\
\frac{\partial H}{\partial p_{i}}
\end{array}\right)=\left(\begin{array}{l}
0 \\
0
\end{array}\right) .
$$

\section{Multi Time Fractional Lagrange Equations}

We consider the multi time variable

$$
\left(t^{v}\right)=\left(t^{1}, t^{2}, \ldots, t^{p}\right) \in R^{p}, \quad v=1, \ldots, p .
$$

Let $a=\left(a^{v}\right)$ and $b=\left(b^{v}\right)$ be the borders of the integration and let us consider the functions given below

$$
q^{i}: R^{p} \rightarrow R, \quad\left(t^{1}, t^{2}, \ldots, t^{p}\right) \rightarrow q^{i}\left(t^{1}, t^{2}, \ldots, t^{p}\right), \quad i=1, \ldots, n .
$$

Multi time Lagrangian is considered to be function of left fractional derivatives as

$$
L: R^{p+n+n p} \rightarrow R, \quad\left(t^{\nu}, q^{i},{ }_{a} D_{t^{\nu}}^{\alpha} q^{i}\right) \rightarrow L\left(t^{\nu}, q^{i},{ }_{a} D_{t^{\nu}}^{\alpha} q^{i}\right) .
$$

By making use of the fractional variational principles the corresponding fractional EulerLagrange equations are obtained as follows

$$
\frac{\partial L}{\partial q^{i}}+{ }_{t^{\nu}} D_{b}^{\alpha} \frac{\partial L}{\partial_{a} D_{t^{\nu}}^{\alpha} q^{i}}=0 .
$$

\section{Multi Time Fractional Hamilton Equations}

In 1935 de Donder obtained the multi time Hamilton equation in classical mechanics [7]. Our aim is to obtain the fractional generalization of this result. By using the definition of the fractional momenta as follows

$$
p_{i}^{v}=\frac{\partial L}{\partial_{a} D_{t^{\nu}}^{\alpha} q^{i}}
$$

we define the fractional Hamiltonian as

$$
H={ }_{a} D_{t^{\nu}}^{\alpha} q^{i} \frac{\partial L}{\partial_{a} D_{t^{\nu}}^{\alpha} q^{i}}-L\left(t^{\nu}, q^{i},{ }_{a} D_{t^{\nu}}^{\alpha} q^{i}\right) .
$$


The transformation ${ }_{a} D_{t^{\nu}}^{\alpha} q^{i} \rightarrow p_{i}^{\nu}$ defines the fractional Legendre transformation in multi time case. Taking total differential of (19) and using (18) and canceling two terms we have

$$
d H=-\frac{\partial L}{\partial t^{\nu}} d t^{\nu}-\frac{\partial L}{\partial q^{i}} d q^{i}+{ }_{a} D_{t^{\nu}}^{\alpha} q^{i} d p_{i}^{\nu} .
$$

Taking into account the fractional Euler-Lagrange equation (17) and (18) we obtain

$$
d H=-\frac{\partial L}{\partial t^{\nu}} d t^{\nu}+{ }_{t^{\nu}} D_{b}^{\alpha} p_{i}^{\nu} d q^{i}+{ }_{a} D_{t^{\nu}}^{\alpha} q^{i} d p_{i}^{\nu} .
$$

Equation (21) shows that $H$ is a function of $t^{v}, q^{i}, p_{i}^{v}$. Therefore we can write

$$
d H\left(t^{\nu}, q^{i}, p_{i}^{\nu}\right)=\frac{\partial H}{\partial t^{\nu}} d t^{\nu}+\frac{\partial H}{\partial q^{i}} d q^{i}+\frac{\partial H}{\partial p_{i}^{\nu}} d p_{i}^{\nu} .
$$

Comparing (22) and (21) we obtain

$$
\begin{aligned}
& \frac{\partial H}{\partial t^{\nu}}=-\frac{\partial L}{\partial t^{\nu}}, \quad v=1, \ldots, p, \\
& \frac{\partial H}{\partial q^{i}}={ }_{t^{\nu}} D_{b}^{\alpha} p_{i}^{v}, \quad i=1, \ldots, n, v=1, \ldots, p, \\
& \frac{\partial H}{\partial p_{i}^{v}}={ }_{a} D_{t^{\nu}}^{\alpha} q^{i}, \quad i=1, \ldots, n, v=1, \ldots, p,
\end{aligned}
$$

The number of $(23)$ is $(n+1) p+n$ equations and they are the counterpart of the EulerLagrange equation on $R^{n}$.

\section{Fractional Conservation Laws}

In classical mechanics when Lagrangian is autonomous Hamiltonian is not a constant of motion [6]. In this section we study conservation laws from the existence of symmetries of the fractional variational principles in tow cases.

Case 1. Suppose the functional as follows

$$
I=\int_{a}^{b} L\left(t^{\nu}, q^{i},{ }_{a} D_{t^{\nu}}^{\alpha} q^{i},{ }_{t}{ }^{\nu} D_{b}^{\beta} q^{i}\right) d t
$$

under the appropriate conditions. If (24) is invariant under many parameter group of infinitesimal transformations as

$$
\begin{aligned}
\delta t^{\nu} & =\epsilon_{r} X_{r}^{v}, \quad r=1, \ldots, R, \\
\delta q^{i}(t) & =\epsilon_{r} \psi_{i r}, \quad r=1, \ldots, R,
\end{aligned}
$$

where $R$ is the number of infinitesimal transformation parameters. Using fractional Noethertype theorem [9], the fractional conservation law is

$$
C=\left[\frac{\partial L}{\partial_{a} D_{t^{\nu}}^{\alpha} q^{i}}-\frac{\partial L}{\partial_{t^{\nu}} D_{b}^{\beta} q^{i}}\right] \psi_{i r}+\left[L \delta_{v}^{\mu}-\alpha \frac{\partial L}{\partial_{a} D_{t^{\mu}}^{\alpha} q_{a}^{i}} D_{t^{\nu}}^{\alpha} q^{i}-\beta \frac{\partial L}{\partial_{t^{\mu}} D_{b}^{\beta} q^{i} t^{\nu}} D_{b}^{\beta} q^{i}\right] X_{r}^{v},
$$


in the sense of the following definition [9]

$$
D_{t^{\nu}}^{\alpha}\{(f g)\}=-f \sum_{\mu} t^{\mu} D_{b}^{\alpha} g+g \sum_{\mu}{ }_{a} D_{t^{\mu}}^{\alpha} f
$$

In (26) if we choose $\mu=1$ and $v=1$ we have corresponding conservation law for the case of single time fractional Lagrangian [9].

Equation (27) for multi time classical mechanics becomes divergence in time sense as follows

$$
D(f g)=\sum_{\mu} \frac{\partial}{\partial t_{\mu}}(f g) .
$$

Case 2. Let us consider the following functional

$$
I=\int_{a}^{b} L\left(t^{\nu}, q^{i}, q_{v}^{i}\right) d t
$$

where $q_{\nu}^{i}=\left(\frac{d q^{i}}{d t^{v}}\right)$. Likewise case 1 if we suppose the functional (29) is invariant under the group of infinitesimal transformations then the conservation law is

$$
C=\frac{\partial L}{\partial q_{v}^{i}} \psi_{i r}+\left(L \delta_{v}^{\mu}-\frac{\partial L}{\partial q_{\mu}^{i}} q_{v}^{i}\right) X_{r}^{v}
$$

In (30) let the variations are $\psi_{i r}=0$ and $X_{r}^{\nu}=\delta_{r}^{\nu}$ then

$$
C=T_{r \mu}=L \delta_{r}^{\mu}-\frac{\partial L}{\partial q_{\mu}^{i}} q_{r}^{i},
$$

energy tensor is conservation law.

Let us consider the group of infinitesimal transformations for single time Lagrangian as follows

$$
\delta t=\epsilon_{r} X_{r}, \quad \delta q^{i}=\epsilon_{r} \Psi_{r i}
$$

The corresponding conservation law is

$$
C=\frac{\partial L(t, q, \dot{q})}{\partial \dot{q}^{i}} \psi_{i r}+\left(L-\frac{\partial L(t, q, \dot{q})}{\partial \dot{q}^{i}} \dot{q}^{i}\right) X_{r}
$$

In (32) let $\psi_{i r}=0$ and $X_{r}=\delta_{r 1}$, then the conservation law reduces to Hamiltonian,

$$
H=L-\frac{\partial L(t, q, \dot{q})}{\partial \dot{q}^{i}} \dot{q}^{i}
$$

As we have seen the fractional conservation law in special cases reduces to well known conservation law in classical mechanics. 


\section{Legendre Transform of Fractional Multi Time Hamiltonian Dual Action}

We write the fractional multi time Hamilton equations in the form

$$
\begin{aligned}
& -\delta_{\mu}^{v} \delta_{j^{\nu}}^{i} D_{b}^{\alpha} p_{i}^{\mu}+\frac{\partial H}{\partial q^{j}}=0, \\
& \quad i=1, \ldots, n, j=1, \ldots, n, v=1, \ldots, p, \mu=1, \ldots, p, \\
& -\delta_{\mu}^{v} \delta_{j a}^{i} D_{t^{\nu}}^{\alpha} q^{i}+\frac{\partial H}{\partial p_{j}^{\mu}}=0, \\
& \quad i=1, \ldots, n, j=1, \ldots, n, v=1, \ldots, p, \mu=1, \ldots, p,
\end{aligned}
$$

or in matrix form

$$
-\left(\begin{array}{cc}
0 & \delta_{\mu}^{\nu} \delta_{j}^{i} \\
\delta_{\mu}^{\nu} \delta_{j}^{i} & 0
\end{array}\right)\left(\begin{array}{c}
{ }_{a} D_{t^{\nu}}^{\alpha} q^{i} \\
{ }_{t^{\nu}} D_{b}^{\alpha} p_{i}^{\mu}
\end{array}\right)+\left(\begin{array}{c}
\frac{\partial H}{\partial q^{j}} \\
\frac{\partial H}{\partial p_{j}^{\mu}}
\end{array}\right)=\left(\begin{array}{l}
0 \\
0
\end{array}\right) .
$$

Using the notation

$$
\left(\begin{array}{cc}
0 & \delta_{\mu}^{v} \delta_{j}^{i} \\
\delta_{\mu}^{v} \delta_{j}^{i} & 0
\end{array}\right)=\delta \otimes J
$$

we can write (34) and (35) as follows

$$
-(\delta \otimes J)\left(\begin{array}{c}
{ }_{a} D_{t^{\nu}}^{\alpha} q^{i} \\
{ }_{t^{\nu}} D_{b}^{\alpha} p_{i}^{\mu}
\end{array}\right)+\left(\begin{array}{c}
\frac{\partial H}{\partial q^{j}} \\
\frac{\partial H}{\partial p_{j}^{\mu}}
\end{array}\right)=\left(\begin{array}{l}
0 \\
0
\end{array}\right) .
$$

The action $\Psi$ whose fractional Euler-Lagrange equations are fractional Hamilton equations is

$$
\Psi=\int_{T_{0}} \mathcal{L}\left(q^{i},{ }_{a} D_{t^{\nu}}^{\alpha} q^{i}, p_{i}^{v}, t^{\nu} D_{b}^{\alpha} p_{i}^{v}\right) d t,
$$

where $d t=d t^{1} d t^{2} \cdots d t^{p}$ and $T_{0}=\left[0, T^{1}\right] \times \cdots \times\left[0, T^{p}\right]$,

$$
\begin{aligned}
& \mathcal{L}=-\frac{1}{2}\left(q_{t^{\nu}}^{i} D_{b}^{\alpha} p_{i}^{\nu}+p_{i a}^{\nu} D_{t^{\nu}}^{\alpha} q^{i}\right)+H\left(t^{\nu}, q^{i}, p_{i}^{\nu}\right) \\
& =-\frac{1}{2}\left({ }_{t^{\nu}} D_{b}^{\alpha} p_{j}^{\nu} \quad{ }_{a} D_{t^{\mu}}^{\alpha} q^{j}\right)\left(\begin{array}{cc}
\delta^{i j} & 0 \\
0 & \delta^{\mu \nu} \delta_{i j}
\end{array}\right)\left(\begin{array}{c}
q^{i} \\
p_{i}^{v}
\end{array}\right)+H\left(t^{\nu}, q^{i}, p_{i}^{v}\right) .
\end{aligned}
$$

Since we can write

$$
\left({ }_{t^{\nu}} D_{b}^{\alpha} p_{j}^{\nu} \quad{ }_{a} D_{t^{\mu}}^{\alpha} q^{j}\right)=(\delta \otimes J) D_{t^{\nu}}^{\alpha} u,
$$

and the standard Riemannian metric from $R^{n+n p}$ is

$$
G=\left(\begin{array}{cc}
\delta^{i j} & 0 \\
0 & \delta^{\mu \nu} \delta_{i j}
\end{array}\right)
$$

also if we suppose $u=\left(q^{i} p_{i}^{\nu}\right), D_{t^{\nu}}^{\alpha} u=\left({ }_{a} D_{t^{\nu}}^{\alpha} q^{i}{ }_{t^{\nu}} D_{b}^{\alpha} p_{i}^{\nu}\right)$ and $\langle$,$\rangle is scalar product and using$ (42), then we have

$$
\mathcal{L}=-\frac{1}{2}\left\langle(\delta \otimes J) D_{t^{\nu}}^{\alpha} u, u\right\rangle+H\left(t^{\nu}, u\right)
$$


We have fractional Lagrange equations as given below

$$
\begin{array}{cc}
\frac{\partial \mathcal{L}}{\partial q^{i}}+{ }_{t^{\nu}} D_{b}^{\alpha} \frac{\partial \mathcal{L}}{\partial_{a} D_{t^{\nu}}^{\alpha} q^{i}}=0, \quad i=1,2, \ldots, n, \\
\frac{\partial \mathcal{L}}{\partial p_{i}^{\nu}}+{ }_{a} D_{t^{\nu}}^{\alpha} \frac{\partial \mathcal{L}}{\partial_{t^{\nu}} D_{b}^{\alpha} p_{i}^{\nu}}=0, \quad i=1,2, \ldots, n .
\end{array}
$$

We obtain the Legendre transformation of $H\left(t^{\nu},.\right)$ as follows

$$
\mathcal{H}^{*}\left(t^{\nu}, \frac{\partial H}{\partial q^{i}}, \frac{\partial H}{\partial p_{i}^{v}}\right)=-q^{i} \frac{\partial H}{\partial q^{i}}-p_{i}^{\nu} \frac{\partial H}{\partial p_{i}^{v}}+H\left(t^{\nu}, q^{i}, p_{i}^{v}\right) .
$$

Substituting Hamilton equations (34) and (35) into (47) we get

$$
\mathcal{H}^{*}\left(t^{\nu},-{ }_{a} D_{t^{\nu}}^{\alpha} q^{i},-{ }_{t^{\nu}} D_{b}^{\alpha} p_{i}^{\nu}\right)=-q_{t^{\nu}}^{i} D_{b}^{\alpha} p_{i}^{\nu}-p_{i a} D_{t^{\nu}}^{\alpha} q^{i}+H\left(t^{\nu}, q^{i}, p_{i}^{\nu}\right) .
$$

By using the definition of scalar product we obtain

$$
\mathcal{H}^{*}\left(t^{\nu},-{ }_{a} D_{t^{\nu}}^{\alpha} q^{i},-{ }_{t^{\nu}} D_{b}^{\alpha} p_{i}^{\nu}\right)=-\left\langle(\delta \otimes J) D_{t^{\nu}}^{\alpha} u, u\right\rangle+H\left(t^{\nu}, q^{i}, p_{i}^{\nu}\right) .
$$

Since we have obtain the Legendre transform of $H\left(t^{\nu},.\right), \mathcal{H}^{*}\left(t^{\nu},.\right)$, we would like to write $\mathcal{L}$ versus $\mathcal{H}$, namely

$$
\begin{aligned}
\mathcal{L} & =\frac{1}{2}\left\langle(\delta \otimes J) D_{t^{\nu}}^{\alpha} u, u\right\rangle-\left\langle(\delta \otimes J) D_{t^{\nu}}^{\alpha} u, u\right\rangle+H\left(t^{\nu}, u\right) \\
& =\frac{1}{2}\left\langle(\delta \otimes J) D_{t^{\nu}}^{\alpha} u, u\right\rangle+\mathcal{H}^{*} .
\end{aligned}
$$

Let us define new variables

$$
v=(Q, P)=\left(-q^{i},-p_{i}^{v}\right),
$$

$\mathcal{L}$ in terms of new variables will be denoted by $\mathcal{L}^{*}$. Therefore, we have the following equalities,

$$
(\delta \otimes J) D_{t^{\nu}}^{\alpha} v=-(\delta \otimes J) D_{t^{\nu}}^{\alpha} u
$$

and

$$
\left\langle(\delta \otimes J) D_{t^{\nu}}^{\alpha} v, v\right\rangle=\left\langle(\delta \otimes J) D_{t^{\nu}}^{\alpha} u, u\right\rangle .
$$

As a result $\mathcal{L}^{*}$ becomes

$$
\mathcal{L}^{*}=\frac{1}{2}\left\langle(\delta \otimes J) D_{t^{\nu}}^{\alpha} v, v\right\rangle+\mathcal{H}^{*}\left(t,(\delta \otimes J) D_{t^{\nu}}^{\alpha} v\right) .
$$

We see that $\mathcal{L}^{*}$ is only function of new variable $v$ and $(\delta \otimes J) D_{t}^{\alpha} v$.

\section{Extremal of Dual Action and Multi Time Hamilton Equations}

The dual action $\Phi$ that is defined on suitable functions uses the following Lagrangian

$$
\mathcal{L}^{*}\left(t, v, D_{t} v\right)=\frac{1}{2}\left\langle(\delta \otimes J) D_{t} v, v(t)\right\rangle+\mathcal{H}^{*}\left(t,(\delta \otimes J) D_{t} v\right) .
$$


Although in classical dual action for periodic functions we have $\Phi(v+c)=\Phi(v)$, in fractional dual action this condition won't be true for periodic solution generally. Since $\int D_{t} c+\int D_{t} v \neq 0$ generally,

$$
\begin{aligned}
\Phi(v+c)= & \int \frac{1}{2}\left\langle(\delta \otimes J) D_{t}(v+c), v(t)+c\right\rangle \\
& +H^{*}\left(t,(\delta \otimes J) D_{t}(v+c)\right) \neq \Phi(v) .
\end{aligned}
$$

In classical mechanics we have $\int \frac{d}{d t} c=0$ because the derivative integer order of constant is zero. Also we have $\int \frac{d}{d t} v=0$ if $v$ be a periodic function. Then we have $\Phi(v+c)=\Phi(v)$.

In classical multi time Hamiltonian there is close connection between the periodical critical points of $\Phi$ and periodical solutions of multi time Hamiltonian and but this connection fails for fractional order derivative for the reason that we mentioned above [6]. But a theorem is still true for fractional case.

Theorem If $v$ be a critical point of the action $\Phi$ and

$$
\begin{aligned}
w(t) & =\nabla \mathcal{H}^{*}\left(t, v^{*}\right), \\
D_{t}^{\alpha} v & =\left(-{ }_{a} D_{t^{\nu}}^{\alpha} q^{i},-{ }_{b} D_{t^{\nu}}^{\alpha} p_{i}^{\nu}\right), \\
v^{*} & =\left(-{ }_{b} D_{t^{\nu}}^{\alpha} p_{i}^{\nu},-{ }_{a} D_{t^{\nu}}^{\alpha} q^{i}\right)=(\delta \otimes J) D_{t}^{\alpha} v=\left(v_{i}^{* v}, v^{* i}\right),
\end{aligned}
$$

then we have

$$
(\delta \otimes J) D_{t}^{\alpha} w=\nabla H(t, w) .
$$

Proof Suppose

$$
w=\left(z^{i}, r_{i}^{\nu}\right), \quad a=\left(a^{\nu}\right), \quad b=\left(b^{\nu}\right), \quad v=1, \ldots, p .
$$

From the definition of dual action we have

$$
v=\left(-q_{i},-p_{i}^{\nu}\right) \quad \text { and } \quad v^{*}=\left(-{ }_{b} D_{t^{\nu}}^{\alpha} p_{i}^{\nu},-{ }_{a} D_{t^{\nu}}^{\alpha} q^{i}\right) .
$$

Then

$$
\mathcal{L}^{*}=\frac{1}{2}\left({ }_{t^{\nu}} D_{b}^{\alpha} p_{i}^{\nu} q^{i}+{ }_{a} D_{t^{\nu}}^{\alpha} q^{i} p_{i}^{\nu}\right)+\mathcal{H}^{*}\left(t,-{ }_{t^{\nu}} D_{b}^{\alpha} p_{i}^{\nu},-{ }_{a} D_{t^{\nu}} q^{i}\right)
$$

If $v=\left(-q^{i},-p_{i}^{\nu}\right)$ is a critical point of the dual action, then $\mathcal{L}^{*}$ satisfies the fractional EulerLagrange equations, namely

$$
\begin{aligned}
& \frac{\partial \mathcal{L}^{*}}{\partial q^{i}}+{ }_{t^{\nu}} D_{b}^{\alpha} \frac{\partial \mathcal{L}^{*}}{\partial_{a} D_{t^{\nu}}^{\alpha} q^{i}}=0, \\
& \frac{\partial \mathcal{L}^{*}}{\partial p_{i}^{\nu}}+{ }_{a} D_{t^{\nu}}^{\alpha} \frac{\partial \mathcal{L}^{*}}{\partial_{t^{\nu}} D_{b}^{\alpha} p_{i}^{\nu}}=0 .
\end{aligned}
$$

Substituting (59) into (60) we have

$$
-\frac{1}{2}{ }_{t^{\nu}} D_{b}^{\alpha} p_{i}^{\nu}+{ }_{t^{\nu}} D_{b}^{\alpha}\left(\frac{\partial \mathcal{H}^{*}}{\partial_{a} D_{t^{\nu}}^{\alpha} q^{i}}-\frac{1}{2} p_{i}^{\nu}\right)=0,
$$




$$
{ }_{t^{\nu}} D_{b}^{\alpha}\left(\frac{\partial \mathcal{H}^{*}}{\partial_{a} D_{t^{\nu}}^{\alpha} q^{i}}\right)={ }_{t^{\nu}} D_{b}^{\alpha} p_{i}^{\nu} .
$$

Also, substituting (59) into (61) we have

$$
\begin{aligned}
-\frac{1}{2}{ }_{a} D_{t^{\nu}}^{\alpha} q^{i}+{ }_{a} D_{t^{\nu}}^{\alpha}\left(\frac{\partial \mathcal{H}^{*}}{\partial_{t^{\nu}} D_{b}^{\alpha} p_{i}^{\nu}}-\frac{1}{2} q^{i}\right) & =0, \\
{ }_{a} D_{t^{\nu}}^{\alpha}\left(\frac{\partial \mathcal{H}^{*}}{\partial_{t^{\nu}} D_{b}^{\alpha} p_{i}^{\nu}}\right) & ={ }_{a} D_{t^{\nu}}^{\alpha} q^{i} .
\end{aligned}
$$

Let define new variables as below

$$
\begin{aligned}
z^{i} & =\frac{\partial \mathcal{H}^{*}}{\partial_{a} D_{t^{\nu}}^{\alpha} q^{i}}, \\
r_{i}^{\nu} & =\frac{\partial \mathcal{H}^{*}}{\partial_{t^{\nu}} D_{b}^{\alpha} p_{i}^{\nu}}
\end{aligned}
$$

Applying (66) and (67) in (63) and (65) we get

$$
\begin{aligned}
{ }_{a} D_{t^{\nu}}^{\alpha} z^{i} & ={ }_{a} D_{t^{\nu}}^{\alpha} q^{i}, \\
{ }_{t^{\nu}} D_{b}^{\alpha} r_{i}^{\nu} & ={ }_{t^{\nu}} D_{b}^{\alpha} p_{i}^{\nu} .
\end{aligned}
$$

Since $\omega=\left(z^{i}, r_{i}^{\nu}\right)=\nabla \mathcal{H}^{*}\left(t, v^{* i}, v_{i}^{* \nu}\right)$ and in view of reversibility condition [6] we have

$$
\left({ }_{t^{\nu}} D_{b}^{\alpha} p_{i}^{\nu},{ }_{a} D_{t^{\nu}}^{\alpha} q^{i}\right)=\nabla H(t, \omega) .
$$

Using (68) we have $\left({ }_{t^{\nu}} D_{b}^{\alpha} r_{i}^{\nu},{ }_{a} D_{t^{\nu}}^{\alpha} z^{i}\right)=\nabla H(t, w)$, then we obtain

$$
t^{\nu} D_{b}^{\alpha} r_{i}^{\nu}=\nabla_{z^{i}} H\left(t, z^{i}, p_{i}^{\nu}\right),
$$

and

$$
{ }_{a} D_{t^{\nu}}^{\alpha} z^{i}=\frac{\partial H}{\partial r_{i}^{\nu}},
$$

respectively. If $w=\left(z^{i}, r_{i}^{\nu}\right)$ then

$$
\left({ }_{t^{\nu}} D_{b}^{\alpha} r_{i}^{\nu},{ }_{a} D_{t^{\nu}}^{\alpha} z^{i}\right)=\nabla H(t, w) \quad \text { or } \quad(\delta \otimes J) D_{t^{\nu}}^{\alpha} w=\nabla H(t, w)
$$

as required.

Remark 1 If we change the derivative to Caputo derivative for the derivative of constant will be zero, ${ }_{a}^{c} D_{t}^{\alpha} c=0$ but we still have $\int_{a}^{c} D_{t}^{\alpha} v \neq 0$. May be useful to know the relation between the periodic functions and fractional integrals

$$
{ }_{a} I_{t a}^{\alpha c} D_{t}^{\alpha} f(t)=f(t)-f(a),
$$

where $\alpha \in(0,1]$ and ${ }_{a} I_{t}^{\alpha} f(t)=\frac{1}{\Gamma(\alpha)} \int_{a}^{t}(t-\tau)^{\alpha-1} f(\tau) d \tau$. If $f(t)$ be periodic function in $t \in[a, T]$, we have

$$
{ }_{a} I_{t a}^{\alpha c} D_{T}^{\alpha} f(t)=f(T)-f(a)=0 .
$$

Remark 2 We recovered the classical results for $\alpha$ integers. 


\section{Conclusion}

Fractional mechanics describes both conservative and non-conservative systems. Recently, the fractional variation principles gained importance in studying the fractional mechanics and several versions are proposed. The fractional Lagrangian and Hamiltonian formulation for the constrained systems are still at the beginning of their development. Fractional mechanics is a non-local theory. The fractional derivatives were renormalized to construct local fractional differential operators. Using fractional Caputo derivative in fractional mechanics allows utilization of initial values of classical integer-order derivative with known physical interpretations. In this paper we generalized classical multi time Hamiltonian to fractional multi time Hamiltonian. Constant of motion in fractional multi time Hamiltonian has been introduced. Further we have shown that fractional multi time mechanics leads to the standard cases. Furthermore the dual action is defined for fractional mechanics. We have explained that the connection between the periodical solution of this dual action and fractional Hamiltonian fails for the formalism involving Riemann-Liouville derivatives. All classical results are recovered for $\alpha$ being an integer.

Open Access This article is distributed under the terms of the Creative Commons Attribution Noncommercial License which permits any noncommercial use, distribution, and reproduction in any medium, provided the original author(s) and source are credited.

\section{References}

1. Agrawal, O.P.: Formulation of Euler-Lagrange equations for fractional variational problems. J. Math. Anal. Appl. 272, 368-379 (2002)

2. Baleanu, D., Muslih, S.: Lagrangian, formulation of classical fields within Riemann-Liouville fractional derivatives. Phys. Scr. 72, 119-121 (2005)

3. Baleanu, D., Agrawal, O.P.: Fractional Hamilton formalism within Caputo's derivative. Czech. J. Phys. 56, 1087-1092 (2006)

4. Baleanu, D., Muslih, S., Tas, K.: Fractional Hamiltonian analysis of higher order derivatives systems. J. Math. Phys. 47, 103503 (2006)

5. Baleanu, D., Golmankhaneh Alireza, K., Golmankhaneh Ali, K.: Fractional Nambu mechanics. Int. J. Theor. Phys. 48, 1044 (2009)

6. Constantina, U., Iulian, D.: Periodical solutions of multi time Hamilton equations. Anal. Univ. Bucuresti 55, 179-188 (2005)

7. De Donder, T.: Theorie invariative du des variations (1935)

8. Gorenflo, R., Mainardi, F.: Fractional Calculus: Integral and Differential Equations of Fractional Orders, Fractals and Fractional Calculus in Continuum Mechanics. Springer Verlag, New York (1997)

9. Frederico, G.S.F., Torres, D.F.M.: A formulation of Noether's theorem for fractional Problems of calculus of variations. J. Math. Anal. Appl. 334, 834-846 (2007)

10. Hilfer, R.: Application of Fractional Calculus in Physics. World Scientific, Singapore (2000)

11. Kilbas, A.A., Srivastava, H.H., Trujillo, J.J.: Theory and Applications of Fractional Differential Equations. Elsevier, Amsterdam (2006)

12. Klimek, K.: Fractional sequential mechanics-models with symmetric fractional derivative. Czech. J. Phys. E 51, 1348-1354 (2001)

13. Klimek, K.: Lagrangean and Hamiltonian fractional sequential mechanics. Czech. J. Phys. E 52, 12471253 (2002)

14. Laskin, N.: Fractional quantum mechanics. Phys. Rev. E 62, 3135-3145 (2000)

15. Miller, K.S., Ross, B.: An Introduction to the Fractional Integrals and Derivatives-Theory and Application. Wiley, New York (1993)

16. Muslih, S., Baleanu, D.: Hamiltonian formulation of systems with linear velocities within RiemannLiouville fractional derivatives. J. Math. Anal. Appl. 304, 599-606 (2005)

17. Oldham, K.B., Spanier, J.: The Fractional Calculus. Academic Press, New York (1974)

18. Podlubny, I.: Fractional Differential Equations. Academic Press, New York (1999) 
19. Rabei, E.M., Nawafleh, K.I., Hiijawi, R.S., Muslih, S.I., Baleanu, D.: The Hamilton formalism with fractional derivatives. J. Math. Anal. Appl. 327, 891-897 (2007)

20. Rabei, E.M., Almayteh, I., Muslih, S.I., Baleanu, D.: Hamilton-Jaccobi formulation of systems with Caputo's fractional derivative. Phys. Scr. 77, 015101 (2007)

21. Rabei, E., Tarawneh, D.M., Muslih, S.I., Baleanu, D.: Heisenberg's equations of motio with fractional derivatives. J. Vib. Control 13, 1239-1247 (2007)

22. Riewe, F.: Nonconservative Lagrangian and Hamiltonian mechanics. Phys. Rev. E 53, 1890-1899 (1996)

23. Riewe, F.: Mechanics with fractional derivatives. Phys. Rev. E 55, 3581-3592 (1997)

24. Samko, S.G., Kilbas, A.A., Marichev, O.I.: Fractional Integrals and Derivatives Theory and Applications. Gordon and Breach, New York (1993)

25. Tarasov, V.E.: Fractional variation for dynamical systems: Hamilton and Lagrange approaches. J. Phys. 39(26), 8409-8425 (2006)

26. Tarasov, V.E.: Fractional statistical mechanics. Chaos 16, 033108-033115 (2006)

27. Tarasov, V.E., Zaslavsky, G.M.: Fractional Ginzburg-Landau equation for fractal media. Physica A 354 (2005)

28. Udriste, C.: Fram integral to manifold and metrics to potential maps Atti del'Academia Peloritana dei Pericolanti, Class 1 di Scienze Fis. Mat. e Nat., vol. LXXXI-LXXXII, C1A0401008 (2003-2004)

29. West, B.J., Bologna, M., Grigolini, P.: Physics of Fractal Operators. Springer, New York (2003)

30. Zaslavsky, G.M.: Chaos, fractional kinetics, and anomalous transport. Phys. Rep. 371, 461-580 (2002)

31. Zaslavsky, G.M.: Hamiltonian Chaos and Fractional Dynamics. Oxford University Press, Oxford (2005) 